\title{
Dark origin of the quark flavor hierarchy and mixing
}

\author{
Emidio Gabrielliø, ${ }^{1,2,3}$ Carlo Marzo, ${ }^{3}$ Luca Marzola, ${ }^{3}$ and Kristjan Müürsepp ${ }^{4}$ \\ ${ }^{1}$ Dipartimento di Fisica, Theoretical section, Università di Trieste, \\ Strada Costiera 11, I-34151 Trieste, Italy \\ ${ }^{2}$ INFN, Sezione di Trieste, Via Valerio 2, I-34127 Trieste, Italy \\ ${ }^{3}$ NICPB, Rävala 10, Tallinn 10143, Estonia \\ ${ }^{4}$ Institute of Physics, University of Tartu, W. Ostwaldi 1, 50411 Tartu, Estonia
}

(Received 6 January 2020; accepted 27 March 2020; published 10 April 2020)

\begin{abstract}
We provide a dynamical mechanism for the generation of the Cabibbo-Kobayashi-Maskawa matrix in the context of a recently proposed model of flavor. The framework, based on the paradigm that Yukawa couplings are effective low energy couplings generated radiatively by dark sector interactions, is here extended to include a new scalar field which plays the role of a dark flavon. Being singlet under the Standard Model gauge group but charged under the $U(1)$ symmetry of the dark sector, this particle sources new two- and three-loop diagrams that result in off-diagonal Yukawa interactions, thereby providing a simple explanation for the observed structure of the Cabibbo-Kobayashi-Maskawa (CKM) matrix. By using an effective parametrization of these new loop contributions, we show that the CKM matrix elements can be correctly reproduced for perturbative values of the involved couplings. New implications for dark matter phenomenology are briefly outlined.
\end{abstract}

DOI: 10.1103/PhysRevD.101.075019

\section{INTRODUCTION}

The origin of flavor hierarchy, reflected in the elementary fermion mass spectrum, remains one of the big conundrums of Nature. The precise LHC measurements of the Higgs boson couplings to gauge bosons and third generation fermions strongly support the Standard Model (SM) mechanism of fermion mass generation, based on the Higgs Yukawa couplings and the electroweak symmetry breaking (EWSB) [1-3]. The SM, however, does not shine any light on the puzzle concerning the observed span of Yukawa couplings, should this be related to an unknown underlying mechanism rather than the outcome of free parameters not regulated by any symmetry. On top of that, the structure of the Cabibbo-Kobayashi-Maskawa (CKM) matrix measured in the quark weak interactions [4] further complicates the overall picture and seems to call for a fundamental mechanism to implement, at least in part, the minimal flavor violation ansatz.

The large hierarchy in the fermion masses, as well as the absence of any underlying symmetry in the Yukawa sector strongly suggest that these interactions could be effective low energy couplings rather than fundamental parameters.

Published by the American Physical Society under the terms of the Creative Commons Attribution 4.0 International license. Further distribution of this work must maintain attribution to the author(s) and the published article's title, journal citation, and DOI. Funded by SCOAP .
The first attempt in this direction dates back to the FroggatNielsen mechanism [5], which relies on higher dimensional operators involving a progressive number of scalar flavon fields $\phi$ charged under a new $U(1)_{F}$ symmetry. In this scheme, the low-energy SM Yukawa couplings then scale as powers of $(\langle\phi\rangle / \Lambda)^{n}$, where $\langle\phi\rangle$ is the vacuum expectation value (vev) of the corresponding flavon field, $\Lambda$ is the effective cutoff scale, and the exponent $n$ depends on the $U(1)_{F}$ charges of the involved fields. It is presently not clear which concrete models of new physics could implement such a construction, and, given the high dimensionality of the operators required to reproduce the full SM fermion spectrum and mixing, whether the mechanism itself could be eventually tested.

A more recent alternative put forward a different solution to the flavor hierarchy puzzle based on the generation of exponentially spread Yukawa couplings $[6,7]$. In this scenario, the SM is extended to include a dark sector consisting of a set of dark fermions singlet under the SM gauge group. New heavy scalar messenger fields, which instead carry the same internal quantum numbers as quarks and leptons, provide generation-blind interactions that bridge the dark and SM sectors. In this construction, the messengers and the dark fermions are charged under a dark $U(1)_{D}$ gauge symmetry, accompanied by the corresponding massless gauge photon: the dark-photon. Phenomenological implications of this scenario have been analyzed in the context of dark photon searches [8-11] and $Z^{\prime}$ boson physics [12]. 
In more detail, the SM Yukawa couplings are enforced to vanish at the tree-level by using a new symmetry $S$, for instance $S U(2)_{L} \times S U(2)_{R}$ in [7]. The interactions in the mediator sector then induce finite loop contributions that result, after the spontaneous symmetry breaking of the $S$ symmetry, in effective low-energy Yukawa couplings $Y_{i}$ proportional to the corresponding dark fermion masses $M_{i}$. Explicitly, we have $Y_{i} \sim M_{i} / \Lambda_{\text {eff }}$, where $\Lambda_{\text {eff }}$ is an almost universal effective scale. Crucially, a nonperturbative $U(1)_{D}$ dynamics [13] in the dark sector generates the desired exponential spread in dark fermion spectrum, leading in the weak $\alpha_{D}$ coupling regime to $[6,7,13]$

$$
Y_{i} \sim M_{i} \sim \exp \left(-\frac{\gamma}{q_{i}^{2} \alpha_{D}}\right) \text {. }
$$

The fundamental parameters $q_{i}$ in the argument of the exponential are related to the $U(1)_{D}$ dark fermion charges, with $\alpha_{D}$ being the corresponding fine-structure constant. The parameter $\gamma$ is instead related to an anomalous dimension. As a consequence, charges $q_{i}$ of the order $\mathcal{O}(1)$ can easily fit the SM spectrum, possibly including Dirac neutrino masses, explaining in this way the origin of the SM flavor hierarchy. Alternatively, a similar exponential scaling law can be achieved in the strongly coupled $U(1)_{D}$ regime via the Miransky mechanism for chiral symmetry breaking $[7,14]$.

In order to also generate the CKM mixing, the portal interaction between the SM fermions $q_{L / R}^{i}$, dark fermions $Q^{j}$, and messengers fields $S^{j}$ must have the form [15]

$$
\mathcal{L} \sim \hat{g}_{L} \bar{q}_{L}^{i} X_{i j}^{L} Q_{R}^{j} \hat{S}_{L}^{j}+\{L \leftrightarrow R\},
$$

where $\hat{g}_{L, R}$ are the corresponding couplings, $X_{i j}$ is a generic matrix and the sum over flavor $(i, j)$ as well as other internal indices (spin, color) is understood. The misalignment between flavor and mass eigenstates that sources the CKM mixing is then ascribed to the $X$ matrix and the diagonal terms in the Yukawa matrices can be recovered by simply setting $X_{i j} \rightarrow \delta_{i j}$.

On general grounds, the off-diagonal contributions to the $X_{i j}$ terms arise after a misalignment between the mass matrices of dark fermions and quark fields. This observation has been used to investigate new processes related to flavor changing neutral currents [15] and kaon physics [16,17]. In order to reproduce the observed hierarchy in the CKM matrix, it is sufficient that the structure of the $X_{i j}$ matrix obey the minimal flavor violation (MFV) hypothesis: $X_{i j}=\delta_{i j}+\Delta_{i j}$, with the off-diagonal terms $\Delta_{i j} \ll 1$ for $i \neq j$. Indeed, although consistent with observations, this ansatz does not motivate the $\Delta_{i j} \ll 1$ condition and additional ad-hoc constraints must, therefore, be imposed [7].

The main objective of the present paper is to overcome this problem, exploring a new mechanism that dynamically generates off-diagonal terms in the Yukawa couplings which respect the MFV condition by construction and recover the observed CKM matrix structure. Our idea is that these entries must be generated at higher orders in perturbation theory with respect to the diagonal terms, which are still the result of one-loop processes.

To this purpose, we restrict the interaction in Eq. (2) to universal diagonal couplings by setting $X_{i j} \rightarrow \delta_{i j}$, and then generate small off-diagonal contributions via higher order radiative processes. The mechanism is implemented by a new scalar field singlet under the SM gauge group, the dark flavon, that mediates $U(1)_{D}$ charged transitions between the dark fermions or the messenger fields. The off-diagonal terms modeled in $X_{i j}$ then arise via the exchange of dark flavons inside the one-loop diagrams used to generate the diagonal elements. In particular, the off-diagonal terms that connect the first two SM generation of up (or down) quarks, $Y_{12}$, as well as the one between the second and third generation, $Y_{23}$, arise at the two-loop level. The remaining $Y_{13}$, coupling, that connects the first and third generation, arises instead only at the 3-loop level. The obtained loop structure then induces an hierarchy between the Yukawa matrix entries and, in turn, results in the observed CKM matrix structure.

As a first check of the scenario, we introduce an effective parametrization of the obtained Yukawa matrix texture that is afterwards constrained by letting the CKM parameters vary in their observed ranges. Besides, we derive analytical expressions for the same Yukawa entries by evaluating the underlying two and three-loop Feynman diagrams induced by the dark flavon insertions. Then, by matching these theoretical predictions against the results obtained with the effective parametrization, we can detail how the observed quark mixing and flavor hierarchy bound the parameter space of the model.

We also outline a new important implication that dark flavon interactions have on the dark matter phenomenology. In fact, whereas in the original scenario $[7,15]$ the dark fermion decay is forbidden by the $U(1)_{D}$ gauge invariance for the absence of $U(1)_{D}$ charged currents, in the model at hand the presence of the dark flavon allows the heaviest of dark fermions to decay. As a consequence, only the lightest of these states can be a potential candidate for dark matter.

The paper is organized as follows. In Sec. II we summarize the features of the original model and show how it explains the SM flavor hierarchy. Section III is instead dedicated to flavor mixing and contains the main point of the present paper. Here, in fact, we introduce the dark flavon and show how the framework at hand can also explain the quark mixing. In Sec. IV, we briefly discuss the new phenomenological aspects of the model resulting from the novel dark flavon interactions. Our conclusions are presented in Sec. V. 


\section{THEORETICAL FRAMEWORK}

We summarize here the main features of the model at the basis of the present work, originally proposed in [6], or in [7] within the context of left-right (LR) gauge symmetry.

As mentioned in the Introduction, the framework firstly requires a new symmetry to forbid the presence of the usual SM Yukawa operators. In regard to this, we adopt here the $S U(2)_{L} \times S U(2)_{R}$ gauge symmetry group instead of the $Z_{2}$ discrete symmetry originally proposed in [6]. The model therefore includes two Higgs doublets, $H_{L}$ and $H_{R}$, to operate the spontaneous symmetry breaking of the corresponding $S U(2)_{L / R}$ interactions, as well as the gauge bosons of the new $S U(2)_{R}$ group. The usual SM Yukawa interactions then arise as low energy effective operators after the $S U(2)_{R}$ is spontaneously broken.

However, the successful radiative generation of Yukawa couplings still requires the presence of a sector external to the SM, responsible for providing the chiral symmetry breaking that sources the SM fermion masses. This is the so-called dark sector, an hidden sector of the theory put into contact with the SM by a set of messenger fields. In particular, the minimal setup for the dark sector includes ${ }^{1}$

(1) dark fermions: a set of six massive Dirac fermions in one-to one correspondence with one SM quark field. The dark fermions are singlets under the SM gauge interactions (or, in the extended left-right (LR) version, singlet under the $S U(2)_{L} \times S U(2)_{R} \times U(1)_{Y}$ gauge group), but charged under a new, unbroken, $U(1)_{D}$ dark interaction. The presence of an unbroken $U(1)_{D}$ is necessary in order to generate a natural exponential spread of dark fermion masses via nonperturbative dynamics, see $[11,13]$. The dark fermion masses source the required chiral symmetry breaking and, as a result of messenger interactions, shape the mass spectrum of SM fermions via an almost constant rescaling factor.

(2) messengers: a set of scalar fields that interact with dark fermions and SM particles. Due to the SM quark quantum numbers, the minimal content needed for the colored messenger sector is uniquely predicted, and for the $S U(2)_{L} \times S U(2)_{R} \times U(1)_{Y}$ case it is given by

(a) $2 N$ complex scalar $S U(2)_{L}$ doublets: $\hat{S}_{L}^{U_{i}}$ and $\hat{S}_{L}^{D_{i}}$, (b) $2 N$ complex scalar $S U(2)_{R}$ doublets: $\hat{S}_{R}^{U_{i}}$ and $\hat{S}_{R}^{D_{i}}$, where $\hat{S}_{A}^{U_{i}, D_{i}}=\left(\begin{array}{c}S_{A}^{U_{i}, D_{i}} \\ S_{A, 2}^{U_{i}, D_{i}}\end{array}\right)$, with $A=\{L, R\}, N=3$, and $i=1,2,3$ indicating the generation.

\footnotetext{
${ }^{1}$ Because the present papers aims to explain the SM quark mass hierarchy and mixing, for the sake of simplicity we restrict our discussion to the part of the dark sector that regulates these properties. However, we remark that it is possible to employ the proposed mechanism to explain also the lepton masses and mixing by straightforwardly extending the dark sector to the corresponding dark fermions and messenger fields.
}

TABLE I. Spin and quantum numbers of the dark sector content: messenger fields $\left(\hat{S}_{L}^{D_{i}}, \hat{S}_{L}^{U_{i}}, \hat{S}_{R}^{D_{i}}, \hat{S}_{R}^{U_{i}}\right.$, with $\left.i=1,2,3\right)$ and dark fermions $\left(Q^{D_{i}}, Q^{U_{i}}\right.$, with $\left.i=1,2,3\right) . U(1)_{D}$ is the gauge symmetry corresponding to dark photon interactions with associated charges $q_{D_{i}}$ and $q_{U_{i}}$. With respect to the particle content of the SM, the original framework also contains a dark photon, associated to the $U(1)_{D}$ interactions, the gauge bosons of the $S U(2)_{R}$ group and an Higgs doublet, $H_{R}$, which drives the symmetry breaking of the latter. In this work, we have extend the model to include also a dark flavon scalar field, charged under $U(1)_{D}$, which explains the observed quark flavor mixing.

\begin{tabular}{lcccccc}
\hline \hline Fields & Spin & $S U(2)_{L}$ & $S U(2)_{R}$ & $U(1)_{Y}$ & $S U(3)_{c}$ & $U(1)_{D}$ \\
\hline$\hat{S}_{L}^{D_{i}}$ & 0 & 2 & 0 & $1 / 3$ & 3 & $-q_{D_{i}}$ \\
$\hat{S}_{L}^{U_{i}}$ & 0 & 2 & 0 & $1 / 3$ & 3 & $-q_{U_{i}}$ \\
$\hat{S}_{R}^{D_{i}}$ & 0 & 0 & 2 & $1 / 3$ & 3 & $-q_{D_{i}}$ \\
$\hat{S}_{R}^{U_{i}}$ & 0 & 0 & 2 & $1 / 3$ & 3 & $-q_{U_{i}}$ \\
$Q^{D_{i}}$ & $1 / 2$ & 0 & 0 & 0 & 0 & $q_{D_{i}}$ \\
$Q^{U_{i}}$ & $1 / 2$ & 0 & 0 & 0 & 0 & $q_{U_{i}}$ \\
\hline \hline
\end{tabular}

The $L, R$ labels identify the messenger fields which couple to the SM fermions of the corresponding chirality, in complete analogy with the nomenclature of squark fields within supersymmetric theories. The $\hat{S}_{L, R}^{U_{i}, D_{i}}$ fields then carry the same quantum numbers as the SM quarks of chirality $L, R$, and interact with the electroweak gauge bosons and gluons via their covariant derivatives. Notice that messenger fields also carry the same $U(1)_{D}$ charges as the associated dark fermions, hence the $U(1)_{D}$ charge identifies the flavor states.

The free Lagrangian of messenger fields generally comprises four distinct mass terms, corresponding to the 12 massive $\hat{S}_{L, R}^{U_{i}}$ and $\hat{S}_{L, R}^{D_{i}}$ fields. In order to reduce the number of free parameters, it is possible to maximize the symmetries of the theory with the introduction of a global symmetry in the scalar sector. For instance, imposing a global $S U(6)$ symmetry forces the six messengers in the $L$ and $R$ sectors to common mass scales, reducing the number of free mass parameters to two. Alternatively, considering a global $S U(3) \times S U(3)$ allows for different masses in the up and down-type sectors, and thus results in a total of four free parameters. In this work we choose to impose an $S U(6)$ global flavor symmetry on the scalar messenger sector and use parity to further identify the common mass scale in the $L$ and $R$ sector with a universal messenger mass scale.

We summarize in Table I the quantum numbers of the dark fermions and messenger scalar fields.

The scalar mediator interactions responsible for the radiative generation of the diagonal Yukawa entries in the quark sector are contained in the interaction Lagrangian $\mathcal{L}_{\mathrm{MS}}^{I}$ (we refer the reader to [7] for the full expression) 


$$
\begin{aligned}
\mathcal{L}_{\mathrm{MS}}^{I} \supset \hat{g}_{L}\left(\sum_{i=1}^{N}\left[\bar{\psi}_{L}^{i} Q_{R}^{U_{i}}\right] \hat{S}_{L}^{U_{i}}+\sum_{i=1}^{N}\left[\bar{\psi}_{L}^{i} Q_{R}^{D_{i}}\right] \hat{S}_{L}^{D_{i}}\right) \\
+\hat{g}_{R}\left(\sum_{i=1}^{N}\left[\bar{\psi}_{R}^{i} Q_{L}^{U_{i}}\right] \hat{S}_{R}^{U_{i}}+\sum_{i=1}^{N}\left[\bar{\psi}_{R}^{i} Q_{L}^{D_{i}}\right] \hat{S}_{R}^{D_{i}}\right) \\
+\lambda \sum_{i=1}^{N}\left(\tilde{H}_{L}^{\dagger} \hat{S}_{L}^{U_{i}} \hat{S}_{R}^{U_{i}^{\dagger}} \tilde{H}_{R}+H_{L}^{\dagger} \hat{S}_{L}^{D_{i}} \hat{S}_{R}^{D_{i}^{\dagger}} H_{R}\right)+\text { H.c. },
\end{aligned}
$$

where the color and $S U(2)_{L, R}$ contractions are left understood. The $S U(2)_{L, R}$ doublets $\psi_{L, R}^{i}=\left(\begin{array}{c}U_{L, R}^{i} \\ D_{L, R}^{i}\end{array}\right)$ represent here the SM up $(U)$ and down $(D)$ quark fields, $H_{L, R}=\left(\begin{array}{c}H_{L, R}^{ \pm} \\ H_{L, R}^{0}\end{array}\right)$ are the Higgs doublets and $\tilde{H}_{L, R}$ are, as usual, defined as $\tilde{H}_{L, R}=i \sigma_{2} H_{L, R}^{\star}$. The two constants $\hat{g}_{L}$ and $\hat{g}_{R}$ in Eq. (3) are flavor-universal parameters required to have perturbative values $\hat{g}_{L, R}<1$. In the following we also identify $\hat{g}_{L}=\hat{g}_{R}=g_{L R}$ as imposed by the LR symmetry.

We show in the next subsection how the interactions in Eq. (3) give rise to the diagonal Yukawa couplings for the up and down quark fields.

\section{A. The origin of the flavor hierarchy}

After the $S U(2)_{L} \times S U(2)_{R}$ breaking, the free Lagrangians of up and down mediator fields acquire the form

$$
\mathcal{L}_{S}^{0}=\partial_{\mu} \hat{S}^{\dagger} \partial^{\mu} \hat{S}-\hat{S}^{\dagger} M_{S}^{2} \hat{S}
$$

where $\hat{S} \equiv\left(\hat{S}_{L}, \hat{S}_{R}\right)$ and we omit the $U, D$ indices, with the understanding that the discussion proceeds in the same way in both sectors. In order to simplify the notation, we also omit the generation indices carried by the messenger field. In this way we can write the square mass term in the above equation as

$$
M_{S}^{2}=\left(\begin{array}{cc}
m_{L}^{2} & \Delta \\
\Delta & m_{R}^{2}
\end{array}\right),
$$

where each element is implicitly a $3 \times 3$ matrix, in the generation space, proportional to the identity. In the above expression $\Delta=\frac{1}{2} \lambda v_{R} v_{L} v_{L, R}$ and $v_{L, R} / \sqrt{2}$ indicate the vevs of $\hat{H}_{L, R}$, respectively. The term $\Delta$ then parametrizes the leftright scalar mixing and originates after SSB from an interaction term in the Higgs sector $\lambda\left(\hat{H}_{L}^{\dagger} \hat{S}_{L}\right)\left(\hat{S}_{R}^{\dagger} \hat{H}_{R}\right)$, needed to generate the Yukawa couplings—-see [7] for more details.

The interplay between parity and the global $S U(6)$ flavor symmetry imposed on the free Lagrangian of messenger fields forces all the diagonal terms appearing in Eq. (5) to a common mass scale $m_{L}^{2}=m_{R}^{2}=\bar{m}^{2}$, barring radiative corrections. This condition is necessary to ensure that the quark mass hierarchy will be determined solely by the dark fermion dynamics. The $M_{S}^{2}$ matrix in Eq. (5) is then diagonalized via a unitary matrix

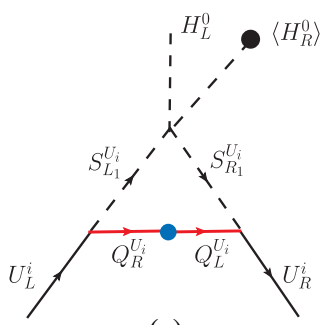

(a)

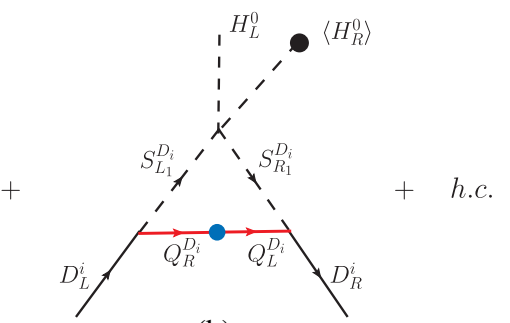

(b)
FIG. 1. Feynman diagrams responsible for the radiative generation of the up-type quarks (a) and down-type quarks (b) Yukawa couplings. The dot on an external Higgs line indicates that the field is set to its vev.

$$
U=\left(\begin{array}{cc}
\cos \theta & \sin \theta \\
-\sin \theta & \cos \theta
\end{array}\right)
$$

with $\tan 2 \theta=\frac{2 \Delta}{m_{L}^{2}-m_{R}^{2}}$. The eigenvalues of the corresponding diagonal mass matrix $M_{S}^{2 \text { diag }}=U M_{S}^{2} U^{\dagger}$ then are

$$
m_{ \pm}^{2}=\frac{1}{2}\left(m_{L}^{2}+m_{R}^{2} \pm\left[\left(m_{L}^{2}-m_{R}^{2}\right)^{2}+4 \Delta^{2}\right]^{1 / 2}\right),
$$

and $m_{L}^{2}=m_{R}^{2}=\bar{m}^{2}$ in our symmetric LR scenario. The $U$ matrix elements consequently simplify to $U(i, i)=1 / \sqrt{2}$, $U(1,2)=-U(2,1)=1 / \sqrt{2}$, yielding square-mass eigenvalues

$$
m_{ \pm}^{2}=\bar{m}^{2}(1 \pm \xi)
$$

where the mixing parameter $\xi$ is given by

$$
\xi=\frac{\lambda v_{R} v_{L}}{2 \bar{m}^{2}} .
$$

The diagonal entries $Y_{f}$ of the SM Yukawa matrices, are then generated through the diagrams presented in Fig. 1, which at low energies give rise to the dimension 5 operator

$$
\mathcal{L}_{\text {eff }}=\frac{1}{\Lambda_{\text {eff }}^{f}}\left(\bar{\psi}_{L}^{f} H_{L}\right)\left(H_{R}^{\dagger} \psi_{R}^{f}\right)+\text { H.c. },
$$

after the replacement $\hat{H}_{R} \rightarrow v_{R}$. We indicated here with $\left(\psi_{L}^{f}\right) H_{L}$ and $\left(\psi_{R}^{f}\right) H_{R}$ the relevant (fermion) Higgs doublets belonging to the indicated chiral sector. The effective constant $\Lambda_{\text {eff }}^{f}$ is determined by matching the matrix elements obtained from the effective low energy operator in Eq. (10) with the corresponding expressions in the fundamental theory, given by the computation of the one-loop diagram in Fig. 1. The resulting expression for $Y_{f}$ as a function of the mixing $\xi$ is [15]

$$
Y_{f}=\left(\frac{g_{L R}^{2}}{16 \pi^{2}}\right)\left(\frac{\xi m_{Q^{f}} \sqrt{2}}{v_{L}}\right) f_{1}\left(x_{f}, \xi\right),
$$



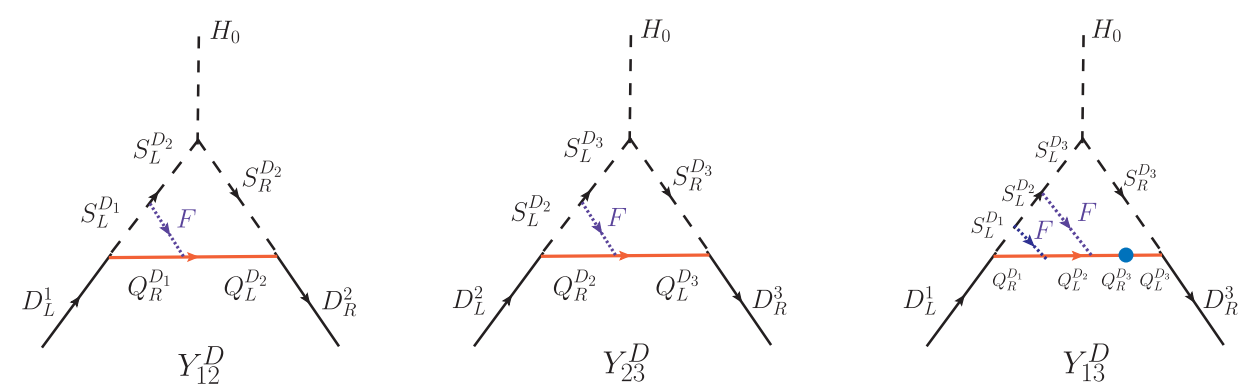

FIG. 2. Diagrams responsible for the radiative generation of the off-diagonal terms in the Yukawa couplings for the down-type quarks. An external $\left\langle H_{R}\right\rangle$ vacuum expectation value insertion at the Higgs vertex is understood. Similar diagrams source the off-diagonal terms in the up-type quark Yukawa matrix.

where $m_{Q^{f}}$ is the mass of the dark fermion $Q^{f}, x_{f}=m_{Q^{f}}^{2} / \bar{m}^{2}$, and the loop function $f_{1}(x, \xi)$ is given by [15]

$f_{1}(x, \xi)=\frac{1}{2}\left[C_{0}\left(\frac{x}{1-\xi}\right) \frac{1}{1-\xi}+C_{0}\left(\frac{x}{1+\xi}\right) \frac{1}{1+\xi}\right]$,

with

$$
C_{0}(x)=\frac{1-x(1-\log x)}{(1-x)^{2}}
$$

The dark fermion masses $m_{Q^{f}}$ can be generated nonperturbatively in the dark sector via the Lee-Wick mechanism for chiral symmetry breaking [13]. Due to the presence of the higher-derivative Lee-Wick term in the $U(1)_{D}$ gauge sector [18-20], the dark fermions acquire masses given by [6]

$$
m_{Q^{f}}=\Lambda \exp \left(-\frac{\gamma}{q_{f}^{2} \alpha_{D}}\right)
$$

where $q_{f}$ are the different $\mathcal{O}(1) U(1)_{D}$ charges of dark fermions and $\gamma$ is a constant connected to an anomalous dimension. The scale $\Lambda$ refers to the scale in the Lee-Wick term of the $U(1)_{D}$ gauge sector. By inserting Eq. (14) into (11), we can then see that the hierarchy in the diagonal components of the obtained Yukawa coupling matrix simply reflects the exponential spread of the dark fermion masses.

\section{THE ORIGIN OF THE CKM MATRIX}

In the previous section we discussed the generation of hierarchical diagonal terms in the Yukawa matrix. In order to fully address the problem of flavor, we now need to address the remaining off-diagonal entries, which are responsible for inducing the quark mixing modeled in the CKM matrix.

These terms were modelled in the original framework by generalizing the interactions in Eq. (3) to a mixing between quarks of generation $i$ and dark fermions and messengers of generation $j$ [7], postulated to be small in accordance with the MFV hypothesis. In order to address this shortcoming, in the following we detail a new dynamical mechanism to generate suppressed off-diagonal terms in the Yukawa matrix. We then introduce a complex single scalar field $F$, the dark flavon, which transforms under $U(1)_{D}$ with a charge $q_{F}$. Moreover, we assume that the $U(1)_{D}$ charges of the dark fermions satisfy the following relations

$q_{F}=q_{2}^{U}-q_{1}^{U}=q_{3}^{U}-q_{2}^{U}=q_{2}^{D}-q_{1}^{D}=q_{3}^{D}-q_{2}^{D}$,

where $q_{i}^{U, D}$ correspond to the $U(1)_{D}$ charges of the dark fermion $Q^{i}$ associated to the quark generation $i$ in the up $(U)$ and down $(D)$ sectors, respectively. It then follows that $q_{2}^{U, D}=\left(q_{3}^{U, D}+q_{1}^{U, D}\right) / 2$, and gauge invariance imposes analogous relations for the $U(1)_{D}$ charges of messenger fields $S_{L_{i}}^{U, D}{ }^{2}$

With this setup, the Lagrangian of the model can be extended to the following interactions that source the off diagonal terms in the Yukawa matrices of up and downtype quarks:

$$
\begin{aligned}
\mathcal{L}_{F}= & \frac{1}{2}\left(D_{\mu} F\right)^{\dagger} D^{\mu} F-\frac{1}{2} m_{F} F^{\dagger} F \\
& +\left(\eta_{U} \bar{Q}^{U_{1}} Q^{U_{2}}+\eta_{U}^{\prime} \bar{Q}^{U_{2}} Q^{U_{3}}\right) F \\
& +\left(\eta_{D} \bar{Q}^{D_{1}} Q^{D_{2}}+\eta_{D}^{\prime} \bar{Q}^{D_{2}} Q^{D_{3}}\right) F \\
& +\mu_{U}\left(\hat{S}_{L}^{U_{1}^{\dagger}} \hat{S}_{L}^{U_{2}}+\hat{S}_{L}^{U_{2} \dagger} \hat{S}_{L}^{U_{3}}\right) F \\
& +\mu_{D}\left(\hat{S}_{L}^{D_{1} \dagger} \hat{S}_{L}^{D_{2}}+\hat{S}_{L}^{D_{2} \dagger} \hat{S}_{L}^{D_{3}}\right) F+\{L \leftrightarrow R\}+\text { H.c. }
\end{aligned}
$$

where $\mathcal{L}_{0}(F)$ is the free Lagrangian of the dark flavon field $F$.

By using the interactions in Eq. (16), we can generate the desired terms via the single or double exchange of the $F$-field in the same one-loop diagram responsible for the diagonal terms, as shown in Fig. 2. The resulting offdiagonal components are then naturally smaller than the diagonal elements, and consequently induce only negligible

\footnotetext{
${ }^{2}$ Notice that the relations in Eq. (15) could be satisfied by simply considering charges taken from a sequence of integer numbers.
} 
corrections to the mass hierarchy regulated by the latter. We anticipate that at least two different couplings, $\eta_{U, D}$ and $\eta_{U, D}^{\prime}$ of the $F$ field to the dark fermions (or, alternatively, to the messenger fields), are needed in order to source the $C P$ violating phase in the resulting CKM matrix.

Following the structure of the diagrams in Fig. 2, we can see that the Yukawa matrices generated are necessarily Hermitian and possess a well defined structure, given in terms of the effective parameters by

$$
\frac{Y^{U}}{y_{t}} \sim\left(\begin{array}{ccc}
A_{r}^{U} \rho_{u} & \epsilon_{1}^{U} & A^{U} \epsilon_{1}^{U} \epsilon_{2}^{U} \\
\epsilon_{1}^{U_{*}} & \rho_{c} & \epsilon_{2}^{U} \\
A^{U} \epsilon_{1}^{U_{*}} \epsilon_{2}^{U_{*}} & \epsilon_{2}^{U_{*}} & 1
\end{array}\right),
$$

and analogously for the down-type Yukawa

$$
\frac{Y^{D}}{y_{b}} \sim\left(\begin{array}{ccc}
A_{r}^{D} \eta_{d} & \epsilon_{1}^{D} & A^{D} \epsilon_{1}^{D} \epsilon_{2}^{D} \\
\epsilon_{1}^{D_{*}} & \eta_{s} & \epsilon_{2}^{D} \\
A^{D} \epsilon_{1}^{D_{*}} \epsilon_{2}^{D_{*}} & \epsilon_{2}^{D_{*}} & 1
\end{array}\right),
$$

where $\rho_{i}=m_{i} / m_{t}, \eta_{i}=m_{i} / m_{b}$, whereas $y_{b}$ and $y_{t}$ are the bottom and top quark SM Yukawa couplings. The terms $A^{D, U}$ and $A_{r}^{D, U}$ are real coefficients of order $\mathcal{O}(1-10)$ and $\mathcal{O}(0-1)$, respectively, while the loop contributions are modeled in the coefficients $\left|\epsilon_{1,2}^{U, D}\right| \ll 1$. The coefficient $A^{D, U}$, in particular, is meant to compensate extra loop and coupling suppression obtained in modeling a three-loop contribution as a product of two two-loop contributions. Differently, the factor $A_{r}^{D, U}$ weights the contribution of quark mixing into the first generation quark masses. With the expressions above, we can then compute the CKM matrix as

$$
V_{\mathrm{CKM}}=V^{U}\left(V^{D}\right)^{\dagger}
$$

where $V^{U}$ and $V^{D}$ are the unitary matrices that diagonalize the corresponding Yukawa matrices

$$
Y_{\mathrm{diag}}^{x}=V^{x} Y^{x}\left(V^{x}\right)^{\dagger}
$$

with $x=U, D$.

The value of the parameters and the phases in the two unitary matrices can then be determined by matching the obtained CKM matrix elements with the corresponding experimental values.

\section{A. Matching the observed quark mixing and hierarchy}

In order to make contact with measurements we perform a numerical scan of the resulting CKM matrix, selecting the
TABLE II. The effective parameter space selected after a preliminary scan.

\begin{tabular}{lcccc}
\hline \hline Parameter & $\begin{array}{c}\text { Lower } \\
\text { bound }\end{array}$ & $\begin{array}{c}\text { Upper } \\
\text { bound }\end{array}$ & Sampling & $\begin{array}{c}\text { Number } \\
\text { of points }\end{array}$ \\
\hline$A_{r}^{D}$ & $10^{-5}$ & 10 & $\exp _{10}$ & 200 \\
$\epsilon_{1}^{D}$ & $10^{-2.5}$ & $10^{-2}$ & $\exp _{10}$ & 200 \\
$\epsilon_{2}^{D}$ & $10^{-1.5}$ & $10^{-1}$ & $\exp _{10}$ & 200 \\
$A^{D}$ & 5 & 45 & lin & 200 \\
\hline \hline
\end{tabular}

parameters that reproduce the observed quark mixing and mass hierarchy.

In this first analysis we simplify our task by neglecting all phases, which can be straightforwardly matched by arranging the relative phases of the complex parameters that enter the diagrams discussed in the previous section. Furthermore, we assume that the Lagrangian of the model is already given on a field basis where the up-type quark Yukawa couplings are diagonal. This choice introduces mixing factors in the interactions of up-type quarks with mediators and dark fermions, but noticeably simplifies our investigation that now needs to keep track of the effective parameters in $Y^{D}$ only.

We report in Table II the ranges of the relevant parameters considered in the analysis, selected after a preliminary scan. For every combination of the parameters drawn from these intervals, we diagonalize the resulting Yukawa coupling matrix and obtain the masses of the down-quark masses upon multiplication by a factor of $m_{b}$, as well as the mixing angle of the CKM matrix. We then compare our findings to the corresponding experimental values, retaining only the combination of input parameters that result in observables within the acceptance limits reported in Table III. For the light quark masses we adopt conservative ranges to account for the uncertainty caused by the running of these parameters. For the remaining quantities, instead, we use the $3 \sigma$ limit from Ref. [21].

The region of the effective parameter space selected by our methodology is presented in Fig. 3. The first three panels highlight the dependence of the selected effective parameters on $\log _{10}\left(A_{r}^{D}\right)$ and reveal the presence of two distinguished solutions, corresponding to $A_{r}^{D}<1$ and

TABLE III. The acceptance criterions used in the scan.

\begin{tabular}{lcc}
\hline \hline Parameter & Lower bound & Upper bound \\
\hline$m_{d}(\mathrm{GeV})$ & $10^{-3}$ & $10^{-2}$ \\
$m_{s}(\mathrm{GeV})$ & $5 \times 10^{-2}$ & $1.5 \times 10^{-1}$ \\
$m_{b}(\mathrm{GeV})$ & 4.06 & 4.3 \\
$\sin \theta_{12}$ & 0.2195 & 0.2291 \\
$\sin \theta_{23}$ & 0.0368 & 0.0458 \\
$\sin \theta_{13}$ & 0.0022 & 0.0052 \\
\hline \hline
\end{tabular}



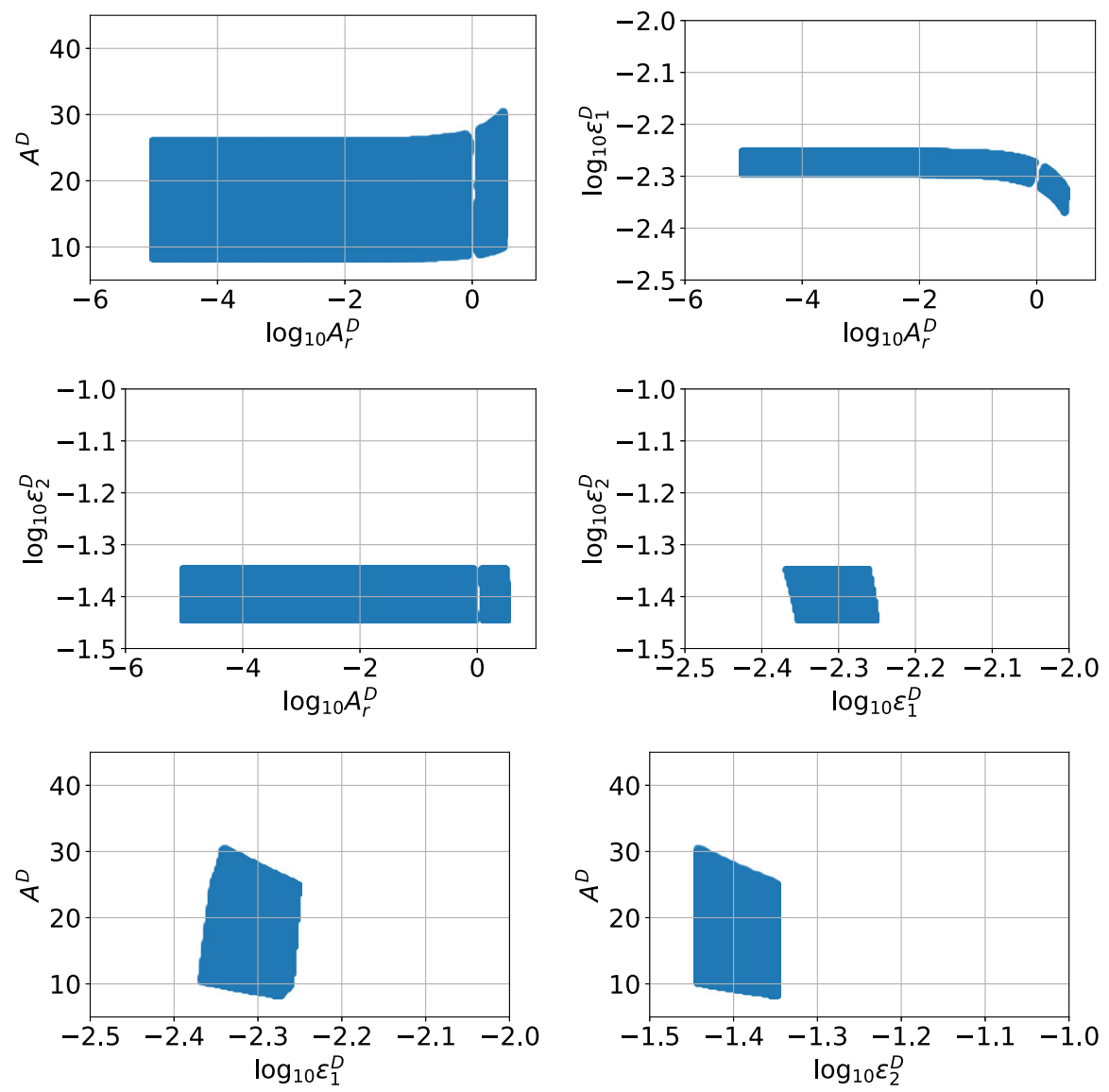

FIG. 3. The combinations of effective input parameters selected by the acceptance intervals given in Table III.

$A_{r}^{D}>1$. In the former case the CKM mixing dominates the contributions into $m_{d}$, whereas for $A_{r}^{D}>1$ this parameter is sourced by the $(1,1)$ component of the Yukawa matrix. The remaining panels show instead the magnitude of the two two-loop level contributions in Fig. 2 required to match the observations, as well as the size of the compensation factor in the employed ansatz for the remaining three-loop contribution.

As for the correlations between effective input parameters and quark sector observables highlighted by our analysis, we present in Fig. 4 the most significative trends. The first panel in the top row shows the dependence of the down quark mass on the $A_{r}^{D}$ parameter, with the two regimes discussed before being clearly visible. In particular, it is evident that the values of $m_{d}$ away from $5 \mathrm{MeV}$ require a nonvanishing $(1,1)$ element in the down quark Yukawa coupling matrix. Likewise, as shown in the second panel, large values of this parameter force the loop contribution that regulates the $(1,2)$ entry to the lowest acceptable values. In the bottom row we show instead the correlation between the mixing angles involving the third generation and the corresponding entries in the CKM matrix. Correctly, the former grows with the magnitude of the latter.

\section{B. Matching with the two and three-loop effective operators from the fundamental theory}

The proposed radiative mechanism naturally presents the propagation of heavy virtual states that mark a clear hierarchy of characteristic mass scales in the model. For this reason, the involved multiloop amplitudes of Fig. 2 can be given a simple analytical form in terms of an expansion in the mass scale ratios entering the considered loop diagrams. To avoid the generation of spurious infrared divergences caused by a naive use of a Taylor expansion inside the virtual momenta integration, we employ a series of theoretical tools developed to such purpose, such as the region expansion [22] or the asymptotic large mass expansion [23]. The latter, in particular, is more suitable for a diagrammatic interpretation in terms of a sum of simpler subdiagrams that, in many cases, are single scale tadpoles. To tackle this theoretical challenge, we employ the Q2E and EXP $[24,25]$ packages, invoked via QGRAF [26] in order to correctly pick the relevant subdiagrams and deal with the momentum distribution accordingly. Finally, all the relevant terms are easily computed via the FORM [27] package MATAD3 [28].

The use of the asymptotic expansion in our matching procedure is easily justified by the zero-momentum limit of 

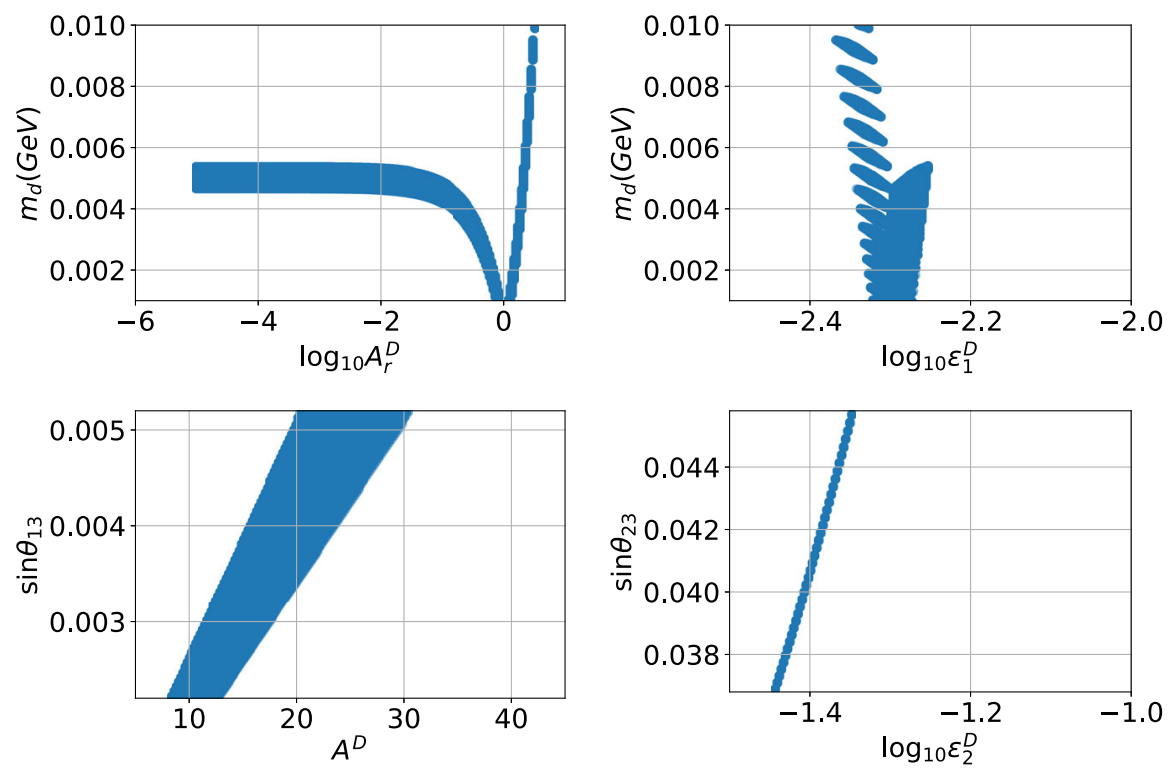

FIG. 4. Selected correlation among the down-quark masses, the CKM parameters and the effective parameters.

the external states, as well as by the choice of three different mass scales associated to the propagating dark flavon (with mass $m_{F}$ ), the dark messenger (characterized, in first approximation, by a common mass scale $m_{S}$ ) and the lightest propagating dark fermion of mass $m_{Q}$. Whereas the requirement that dark fermion constitutes a viable dark matter candidate forces $m_{S}>m_{Q}$, the phenomenology of the scenario does not indicate the ordering of the rest of the spectrum. In particular, although the involved loop functions are certainly sensitive to the relative hierarchy between dark flavons and messengers, a priori we have no reasons to discriminate between the cases $m_{F} \lessgtr m_{S}$.
In order to show how the diagrams in Fig. 2 depend on the involved scales and loop structure, we specialize our discussion to the case $m_{F}=m_{S}$ and use a unique parameter $\chi$ to address all the dimensionless couplings that appear in Eqs. (16) and (3). The trilinear interaction between flavon and messengers is instead parametrized as $\mu_{D}=\rho m_{F}$.

In this way, the result of the asymptotic expansion can be expressed in terms of an overall heavy scale $m_{F}=m_{S}$, the ratio $x_{Q}=m_{Q} / m_{F}$, and of the loop suppression factor $L=16 \pi^{2}$. The number of terms involved grows quickly already after the first leading order contribution. For the sake of completeness, we give here the expansion up to the eighth order in $x_{Q}$ as provided by MATAD3

$$
\begin{aligned}
\frac{m_{F}}{v_{R}} \frac{L^{2}}{\chi^{4} \rho} Y_{12}^{D} \sim \frac{m_{F}}{v_{R}} \frac{L^{2}}{\chi^{4} \rho} Y_{23}^{D}= & \frac{1}{12}\left(-27 S_{2}+\pi^{2}-6\right)+\frac{1}{4}\left(45 S_{2}-\pi^{2}+2\right) x_{Q}^{2} \\
& +x_{Q}^{4}\left(45 S_{2}+\frac{11 \log \left(x_{Q}\right)}{3}-\frac{\pi^{2}}{2}-\frac{179}{36}\right) \\
& +x_{Q}^{6}\left(99 S_{2}+4 \log ^{2}\left(x_{Q}\right)+\frac{353 \log \left(x_{Q}\right)}{30}-\frac{\pi^{2}}{3}-\frac{17353}{900}\right) \\
& +x_{Q}^{8}\left(\frac{693 S_{2}}{4}+18 \log ^{2}\left(x_{Q}\right)+\frac{976 \log \left(x_{Q}\right)}{35}+\frac{3 \pi^{2}}{4}-\frac{911367}{19600}\right)+O\left(x_{Q}^{10}\right)
\end{aligned}
$$

and an analogous expression holds for $Y_{23}^{D}$. For the three-loop function we have instead 


$$
\begin{aligned}
\frac{m_{F}}{v_{R}} \frac{L^{3}}{\chi^{5} \rho^{2}} Y_{13}^{D}= & -\frac{1}{24} x_{Q}\left(459 S_{2}-112 \zeta(3)+\pi^{2}\right)+-x_{Q}^{3} \frac{\left(-9120 O_{\epsilon}^{S_{2}}+15\left(196992 S_{2}+912 T_{\epsilon}^{1}-45432 \zeta(3)+7655\right)\right)}{8640} \\
& -x_{Q}^{3} \frac{\left(4320\left(-27 S_{2}+\pi^{2}-6\right) \log \left(x_{Q}\right)+361 \pi^{4}+30165 \pi^{2}\right)}{8640} \\
& -x_{Q}^{5} \frac{\left(-98400 O_{\epsilon}^{S_{2}}+1080\left(846 S_{2}+12 \pi^{2}-203\right) \log \left(x_{Q}\right)\right)}{38880} \\
& -x_{Q}^{5} \frac{\left(27102114 S_{2}+147600 T_{\epsilon}^{1}-4948920 \zeta(3)+3895 \pi^{4}+228981 \pi^{2}+782769\right)}{38880} \\
& +x_{Q}^{7} \frac{\left(-175778400 O_{\epsilon}^{S_{2}}+60480 \log \left(x_{Q}\right)\left(-224325 S_{2}-10260 \log \left(x_{Q}\right)+2565 \pi^{2}+11234\right)\right)}{24494400} \\
& +x_{Q}^{7} \frac{\left(-1252502190 S_{2}+263667600 T_{\epsilon}^{1}-3233475000 \zeta(3)+6957895 \pi^{4}\right)}{24494400} \\
& +x_{Q}^{7} \frac{\left(740318925 \pi^{2}+3458071731\right)}{24494400}+O\left(x_{Q}^{9}\right)
\end{aligned}
$$

In both the above expressions $v_{R} / \sqrt{2}$ indicates the vev of $H_{R}$ and the explicit form for the $S_{2}, T_{\epsilon}^{1}$ and $O_{\epsilon}^{S_{2}}$ functions are explicitly given below [28]:

$$
\begin{aligned}
S_{2} & =\frac{4}{9 \sqrt{3}} \mathrm{Cl}_{2}\left(\frac{\pi}{3}\right) \\
O_{\epsilon}^{S_{2}} & =-\frac{763}{32}-\frac{9 \pi \sqrt{3} \ln ^{2} 3}{16}-\frac{35 \pi^{3} \sqrt{3}}{48}+\frac{195}{16} \zeta_{2}-\frac{15}{4} \zeta_{3}+\frac{57}{16} \zeta_{4}+\frac{45 \sqrt{3}}{2} \mathrm{Cl}_{2}\left(\frac{\pi}{3}\right)-27 \sqrt{3} \operatorname{Im}\left[\operatorname{Li}_{3}\left(\frac{e^{-i \pi / 6}}{\sqrt{3}}\right)\right], \\
T_{\epsilon}^{1} & =-\frac{45}{2}-\frac{\pi \sqrt{3} \ln ^{2} 3}{8}-\frac{35 \pi^{3} \sqrt{3}}{216}-\frac{9}{2} \zeta_{2}+\zeta_{3}+6 \sqrt{3} \mathrm{Cl}_{2}\left(\frac{\pi}{3}\right)-6 \sqrt{3} \operatorname{Im}\left[\operatorname{Li}_{3}\left(\frac{e^{-i \pi / 6}}{\sqrt{3}}\right)\right],
\end{aligned}
$$

where $\mathrm{Cl}_{2}(x)=\operatorname{Im}\left[\operatorname{Li}_{2}\left(e^{i x}\right)\right]$.

We use the above expression to evaluate the two-loop contribution, shown in Figure 5 as a function of the $A^{D}$ and $m_{Q} / m_{F}$ for different values of $\rho=\mu_{D} / m_{F}$. In order to make contact with the effective parametrization adopted above, we have rescaled the obtained values by the bottom quark Yukawa coupling and have set $\chi=1 / 2$ to ensure perturbativity. Two-loop contributions of the size indicated by the third panel in Fig. 2 then require the scale of the dark fermions to be close to that of the mediators, as well as sizable values of the trilinear coupling of the dark flavon to the messengers. As for the three-loop contribution, the requirement $Y_{13}^{D}=A^{D} Y_{12}^{D} Y_{23}^{D}$ is always satisfied via a suitable choice of the $m_{F} / v_{R}$ ratio, regardless of the mass spectrum chosen for the particles in the dark sector. We also checked that introducing a further hierarchy between messengers and dark flavon does not appear to substantially modify the results of our analysis. However we choose not to rely on these results as the computational effort required to reach a comparable precision is too demanding.

In conclusion, the performed analysis reveals that the Yukawa coupling texture predicted by the framework can indeed match the observed quark mass hierarchy and mixing for a suitable choice of the free parameters of the model. In regard of this, we find that the dimensionless couplings in Eqs. (16) and (3) are allowed to fall well within the perturbative regime. The required size of the two-loop contribution, instead, forces the dark fermion mass scale close to that of messenger and flavon fields and imposes sizeable values of the dark flavon-messengers trilinear coupling that, however, do not undermine perturbative unitarity.

\section{NOVEL PHENOMENOLOGICAL IMPLICATIONS}

To conclude, we briefly discuss the main phenomenological aspects of the proposed model. Although the signatures of this scenario at collider and low energy experiments match those of the original proposal [8-12, 15-17], the presence of novel dark flavon interactions strongly modifies its possible cosmological implications. In particular, imposing the flavor symmetry in the messenger sector for both up and down sectors, the scenario predicts a dark fermion mass spectrum that matches the SM fermion one upon an almost constant rescaling.

The dark fermion mass spectrum is constrained by vacuum stability arguments and by the requirements of 

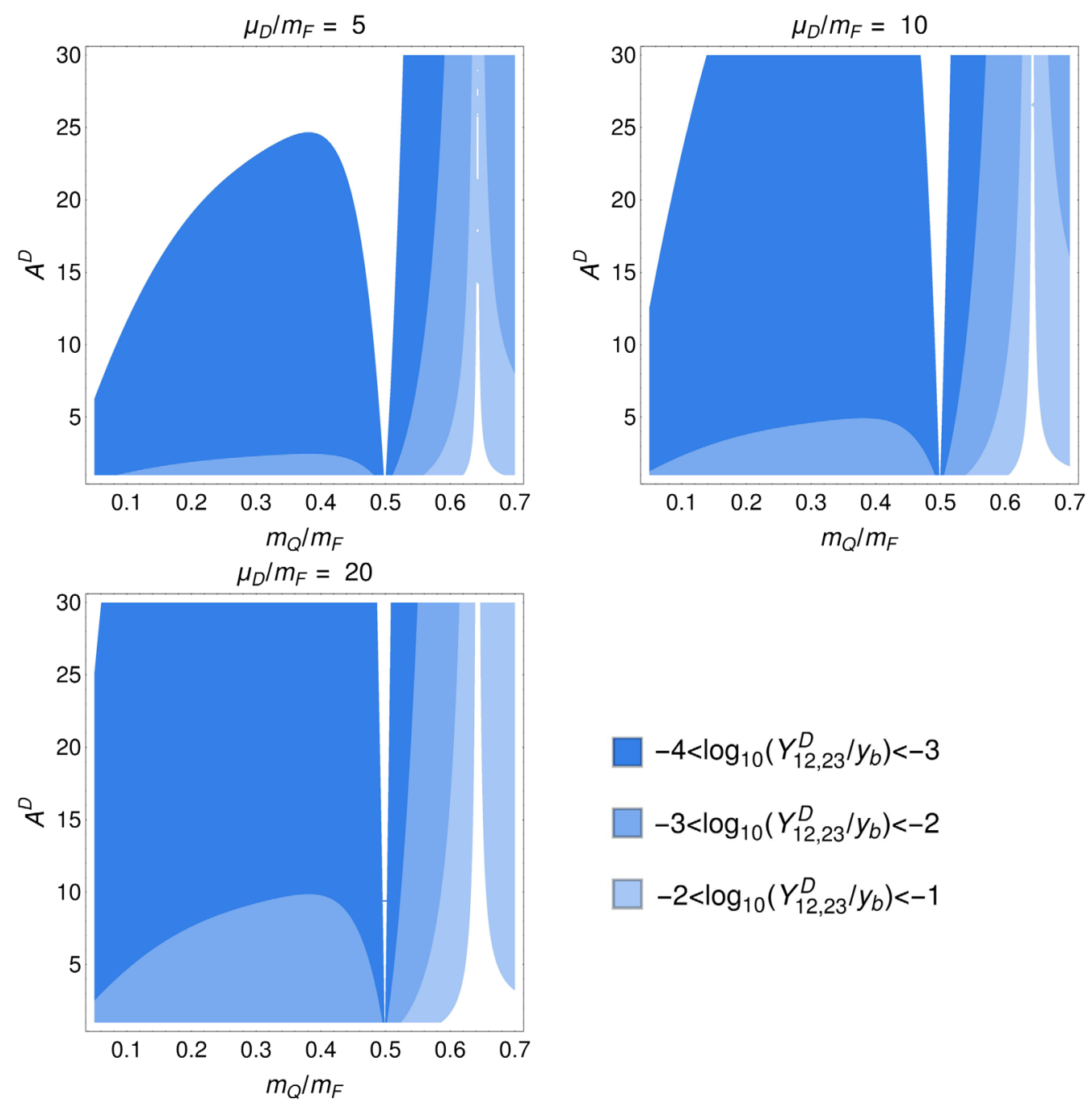

$$
\begin{aligned}
& \square-4<\log _{10}\left(Y_{12,23}^{D} / y_{b}\right)<-3 \\
& \square-3<\log _{10}\left(Y_{12,23}^{D} / y_{b}\right)<-2 \\
& \square-2<\log _{10}\left(Y_{12,23}^{D} / y_{b}\right)<-1
\end{aligned}
$$

FIG. 5. Values of the two-loop function (rescaled by the bottom Yukawa coupling) obtained with the analytical expression in Eq. (21), as a function of the $A^{D}$ parameter and of the mass hierarchy between messengers and dark fermions. All dimensionless coupling constants are set to $1 / 2$, while the dark flavon trilinear coupling $\rho=\mu_{D} / m_{F}$ is set at the reported values.

dark matter, which set the heaviest dark fermion mass (associated with the top quark) in the ballpark of 50 $100 \mathrm{TeV}[6,7,15]$. As a result, the masses of these particles span from a few $\mathrm{GeV}$ up to the $\mathrm{TeV}$ scale, with an even lighter spectrum component allowed for dark fermions associated with the SM lepton sector. We remark that whereas the $U(1)_{D}$ gauge invariance and the absence of corresponding charged currents forbid all dark fermion decays in the original model, the considered flavon interactions enable these processes in the present framework. This is an important difference in the phenomenological consequences of the two scenarios, which attributes novel and richer signatures to the model at hand. In fact, the tree-level exchange of a heavy dark flavon $F$ induces an effective 4-fermion interaction of the form

$$
\mathcal{L}_{\text {eff }} \sim \frac{\eta_{A} \eta_{B}^{\prime}}{\Lambda_{F}^{2}}\left[\bar{Q}^{A_{i}} Q^{A_{i-1}}\right]\left[\bar{Q}^{B_{k}} Q^{B_{k-1}}\right],
$$

where $\Lambda_{F}$ is a scale of the order of the flavon mass, $i, k \in$ $\{1,2,3\}$ and $A, B \in\{U, D\}$. Given the hierarchical mass spectrum of dark fermions, the dark fermions associated with the third SM generation can therefore decay into lighter states. If we assume that each up sector dark fermion is heavier than the corresponding down sector particle, as suggested by the SM Yukawa hierarchy, the $U(1)_{D}$ charges in Eq. (15) allow the following processes

$$
Q^{U_{3}} \rightarrow Q^{U_{2}}+Q^{U_{2}}+\bar{Q}^{U_{1}},
$$

followed by the $Q^{U_{2}}$ decay

$$
Q^{U_{2}} \rightarrow Q^{U_{1}}+Q^{D_{2}}+\bar{Q}^{D_{1}},
$$

provided that the mass relation $m_{Q^{U_{2}}}-m_{Q^{U_{1}}}>2 m_{Q^{D_{2}}}$ hold. Analogously, the decay $Q^{D_{3}} \rightarrow Q^{D_{2}}+Q^{D_{2}}+\bar{Q}^{D_{1}}$ could also be allowed in the dark sector, reducing the 
number of stable dark fermions associated with SM quarks to three: $Q^{U_{1}}, Q^{D_{2}}, Q^{D_{1}}$. The new decay processes mediated by the dark flavon consequently restrict the mass of the heaviest stable dark fermion to the $\mathcal{O}(100 \mathrm{GeV})$ range. In fact, the proposed mechanism relates the dark fermion mass spectrum to that of SM fermions via an almost universal rescaling factor. If the heaviest dark fermion mass is of the order of the common messenger mass in the up sector, the framework indicates a natural upper bound of order $50 \mathrm{TeV}$ for the mass of the dark fermion associated to the top quark [6]. Because of the dark flavon interactions, this particle is allowed to decay into lighter dark fermions. By taking into account the $\mathcal{O}(100)$ ratio between the top and charm quark masses, we expect that the mass of the heaviest stable dark fermion be less than $500 \mathrm{GeV}$.

Remarkably, the presence of the dark flavon is crucial to reconcile the scenario with the theoretical upper bound on the dark matter mass that unitarity of partial waves imposes. In fact, we can expect that the interactions between SM and dark fermion, mediated by the messenger sector, produce a thermal abundance of dark fermions in the early Universe. As the latter expands, the rate of the interactions that keep dark fermions and quarks in thermal equilibrium eventually drops below the Hubble parameter, so the dark fermions decouple from the SM thermal bath and form the dark matter relic abundance observed still today. The yield of the freeze-out mechanism, which regulates this process, is inversely proportional to the thermally averaged annihilation cross section of dark fermions. By decomposing the latter into partial waves subject to the unitarity constraint, it is possible to obtain an upper bound on the annihilation cross section, as well as a corresponding lower bound on the produced relic abundance. As a consequence, for thermally produced dark matter particles not to necessarily over-close the Universe, it must hold that $m_{\mathrm{DM}} \lesssim$ $\mathcal{O}(100) \mathrm{TeV}$ [29]. Whereas the dark fermion associated with the top quark could violate this bound, the dark flavon interactions prevent its stability and therefore indicate the lightest dark state as a suitable dark matter candidate.

Dedicated phenomenological analysis can further constraint the dark fermion mass spectrum, for instance by analyzing the number of thermalized degrees of freedom throughout the different cosmological eras. The dark fermion interactions could also be constrained through indirect detection experiments and cosmic microwave background analysis, although the investigation of these possible signatures requires a dedicated analysis that goes beyond the purpose of the present paper.

As for the collider signatures of the model, the most severe constraints come from the scalar sector. In fact, while the dark fermions properties are not directly testable due to their suppressed interaction with the SM states, the messenger fields must obey the same bounds as squarks of supersymmetric theories. At the LHC, these scalars may be pair produced in QCD interactions and consequently decay into quark and dark fermion at the tree level, resulting in a di-jet and missing energy signature that mimics the squark decays into neutralino and quark. Consequently, it is possible to bound the messenger masses by simply reinterpreting the available squark searches in the limit of gluino decoupling. In our work we have therefore assumed scalar masses well above the current $\mathrm{TeV}$ bound inferred from the LHC limits. However, crucially, within the present framework the quark mixing observables do not depend on the absolute scale of the messenger or and dark flavon fields, but only on their ratio.

\section{CONCLUSIONS}

In the context of models for the radiative generation of SM Yukawa couplings, we have extended the framework originally proposed in [6,7] to accommodate a new mechanism for the origin of flavor mixing. The novelty of our work is in the presence of an additional scalar field, the dark flavon, which sources new loop diagrams that determine the off-diagonal Yukawa matrix elements. The resulting structure recovers the MFV ansatz in a natural way, owing to the additional loop suppression that offdiagonal contributions have with respect to the diagonal ones. The same suppression ensures that the new terms bear a negligible impact on the flavor hierarchy regulated by the diagonal elements, generated here as detailed in the original framework [6,7].

In order to demonstrate the viability of the mechanism, we focused on the flavor hierarchy and mixing that characterize the SM quark sector. In more detail, starting on the flavor basis defined by the up-type quark mass eigenstates, we have introduced an effective parametrization of the down quark Yukawa coupling matrix to model the emerging loop structure. The effective parameter space was then constrained by using the current measurements of the mass spectrum of the down quarks and of the mixing angles contained in the CKM matrix. As a second step, we have evaluated the new two and three-loop diagrams induced by the dark flavon interactions, which source here the off-diagonal Yukawa interactions. By comparing the obtained expressions with the values of the effective parameters selected in the previous step, we have then shown that the current observations can be matched for perturbative values of the involved couplings on top of a mild mass hierarchy in the constituents of the dark sector. Our findings have also highlighted the presence of two qualitatively different solutions to the quark flavor puzzle, related to the emergence of the down quark mass as a pure effect of the quark mixing.

We also remark that the proposed mechanism can be extended to the lepton sector of the SM, although the most straightforward implementation would require the introduction of a further set of corresponding messengers and dark fermion fields. 
The phenomenology of the model scenarios at collider and low energy experiments largely overlaps with that of the original framework. The main difference is that the new dark flavon interactions allow the heaviest dark fermions to decay into the lightest states, reducing the maximal mass of the proposed dark matter candidates. As a consequence, the scenario is fully compatible with the theoretical upper bounds that hold for a relic abundance of elementary particles thermally produced during the evolution of the Universe.

\section{ACKNOWLEDGMENTS}

The authors thank Matthias Steinhauser for providing the Q2E and EXP packages and guidance in their use. The authors are supported by the European Union through the ERDF CoE Grant No. TK133 and by the Estonian Research Council through the Grants No. PRG356 and No. MOBTT86. E. G. is affiliated to the Institute for Fundamental Physics of the Universe, Trieste, Italy.
[1] G. Aad et al. (ATLAS Collaboration), Phys. Lett. B 716, 1 (2012); S. Chatrchyan et al. (CMS Collaboration), Phys. Lett. B 716, 30 (2012).

[2] ATLAS Collaboration, CERN Report No. ATLAS-CONF2019-005, 2019.

[3] A. M. Sirunyan et al. (CMS Collaboration), Eur. Phys. J. C 79, 421 (2019).

[4] M. Tanabashi et al. (Particle Data Group), Phys. Rev. D 98, 030001 (2018).

[5] C. D. Froggatt and H. B. Nielsen, Nucl. Phys. B147, 277 (1979).

[6] E. Gabrielli and M. Raidal, Phys. Rev. D 89, 015008 (2014).

[7] E. Gabrielli, L. Marzola, and M. Raidal, Phys. Rev. D 95, 035005 (2017).

[8] S. Biswas, E. Gabrielli, M. Heikinheimo, and B. Mele, Phys. Rev. D 96, 055012 (2017).

[9] S. Biswas, E. Gabrielli, M. Heikinheimo, and B. Mele, Phys. Rev. D 93, 093011 (2016).

[10] S. Biswas, E. Gabrielli, M. Heikinheimo, and B. Mele, J. High Energy Phys. 06 (2015) 102.

[11] E. Gabrielli, M. Heikinheimo, B. Mele, and M. Raidal, Phys. Rev. D 90, 055032 (2014).

[12] M. Fabbrichesi, E. Gabrielli, and B. Mele, Phys. Rev. Lett. 120, 171803 (2018).

[13] E. Gabrielli, Phys. Rev. D 77, 055020 (2008).
[14] V. A. Miransky, Nuovo Cimento A 90, 149 (1985).

[15] E. Gabrielli, B. Mele, M. Raidal, and E. Venturini, Phys. Rev. D 94, 115013 (2016).

[16] D. Barducci, M. Fabbrichesi, and E. Gabrielli, Phys. Rev. D 98, 035049 (2018).

[17] M. Fabbrichesi, E. Gabrielli, and B. Mele, Phys. Rev. Lett. 119, 031801 (2017).

[18] T. D. Lee and G. C. Wick, Phys. Rev. D 3, 1046 (1971).

[19] T. D. Lee and G. C. Wick, Phys. Rev. D 2, 1033 (1970).

[20] B. Grinstein, D. O'Connell, and M. B. Wise, Phys. Rev. D 77, 025012 (2008).

[21] S. Eidelman et al. (Particle Data Group), Phys. Lett. B 592, 1 (2004).

[22] M. Beneke and V. A. Smirnov, Nucl. Phys. B522, 321 (1998).

[23] V. A. Smirnov, Mod. Phys. Lett. A 10, 1485 (1995).

[24] T. Seidensticker, arXiv:hep-ph/9905298.

[25] R. Harlander, T. Seidensticker, and M. Steinhauser, Phys. Lett. B 426, 125 (1998).

[26] P. Nogueira, J. Comput. Phys. 105, 279 (1993).

[27] B. Ruijl, T. Ueda, and J. Vermaseren, arXiv:1707.06453.

[28] M. Steinhauser, Comput. Phys. Commun. 134, 335 (2001).

[29] K. Griest and M. Kamionkowski, Phys. Rev. Lett. 64, 615 (1990). 